\title{
TURBOGEN: Computer-controlled vertically oscillating grid system for small-scale turbulence studies on plankton
}

\author{
Alberto Amato, ${ }^{1, a)}$ Stefania Fortini, ${ }^{2, a)}$ Romain Watteaux, ${ }^{1}$ Marcello Diano, ${ }^{3}$ Stefania Espa, ${ }^{2}$ \\ Serena Esposito, ${ }^{3}$ Maria I. Ferrante, ${ }^{1}$ Francesc Peters,${ }^{4}$ Daniele ludicone, ${ }^{1, b)}$ \\ and Maurizio Ribera d'Alcalà ${ }^{1, c)}$ \\ ${ }^{1}$ Stazione Zoologica Anton Dohrn, Villa Comunale, 80121 Naples, Italy \\ ${ }^{2}$ Dipartimento di Ingegneria Civile, Edile e Ambientale, Università di Roma, Via Eudossiana, \\ 18, 00184 Rome, Italy \\ ${ }^{3}$ M2M Engineering, Via Cintia Parco S. Paolo, 13, 80125 Naples, Italy \\ ${ }^{4}$ Institut de Ciències del Mar, Passeig Marítim de la Barceloneta, 37-49, Barcelona, Catalunya E-08003, Spain
}

(Received 19 July 2015; accepted 14 March 2016; published online 25 March 2016)

\begin{abstract}
In recent years, there has been a renewed interest in the impact of turbulence on aquatic organisms. In response to this interest, a novel instrument has been constructed, TURBOGEN, that generates turbulence in water volumes up to $13 \mathrm{l}$. TURBOGEN is fully computer controlled, thus, allowing for a high level of reproducibility and for variations of the intensity and characteristics of turbulence during the experiment. The calibration tests, carried out by particle image velocimetry, showed TURBOGEN to be successful in generating isotropic turbulence at the typical relatively low levels of the marine environment. TURBOGEN and its sizing have been devised with the long-term scope of analyzing in detail the molecular responses of plankton to different mixing regimes, which is of great importance in both environmental and biotechnological processes. () 2016 AIP Publishing LLC. [http://dx.doi.org/10.1063/1.4944813]
\end{abstract}

\section{INTRODUCTION}

Turbulence is present in many fluid media and plays an important role in several natural and industrial processes. Devices to generate turbulence within a controlled, predictable, and reproducible intensity range are therefore useful for laboratory experimentation and process studies. In aquatic ecosystems, turbulence strongly affects planktonic organisms over a wide range of scales, ${ }^{1}$ and its impact may substantially affect their function and the related biogeochemical processes.

Peters and Redondo ${ }^{2}$ provided the first exhaustive review of the different devices and experimental setups to generate a turbulent regime suited for plankton studies in laboratory conditions. They stressed that a "good" setup should ensure that the results depend on the variable of choice (e.g., turbulence variation) and not on other drivers (e.g., temperature, nutrients/food availability, or light). In addition, three more requirements were highlighted: ihomogeneous and stationary conditions must be present within the microcosms or mesocosms; ii-turbulence must be isotropic, i.e., invariant with direction; iii-dissipation rates must be as close as possible to the micro-turbulence occurring in the real environment, i.e., in the order of $10^{-9}-10^{-6} \mathrm{~m}^{2} \mathrm{~s}^{-3}$ with a value of $10^{-7} \mathrm{~m}^{2} \mathrm{~s}^{-3}$ in the core of the mixed layer. ${ }^{3}$

Proper characterizations of micro-turbulent fields in nature, especially under high energy conditions, are scanty and mainly restricted to coastal systems, ${ }^{4-7}$ given the difficulty to carry out direct or indirect measurements. ${ }^{8}$ It follows that

\footnotetext{
a) A. Amato and S. Fortini contributed equally to this work.

b)Email: iudicone@szn.it

c) Email: maurizio.ribera@szn.it
}

the main requirements underlined by Peters and Redondo ${ }^{2}$ prioritize the robustness of the experiment vs. the analysis of the effects of a variable turbulent forcing.

Recent field and laboratory observations suggest that turbulence can be inhomogeneous and intermittent also on small spatial and temporal scales, respectively, and even anisotropic in weakly mixed oceanic layers. ${ }^{8}$ The problem of exploring additional solutions for its generation in laboratory devices, suitable to perform experiments in variable and reproducible conditions, is clearly related to that issue.

Traditionally, turbulence is generated mostly by shakers and oscillating grids, ${ }^{2}$ while a completely alternative approach, based on membranes vibrating at high frequency, was proposed a few years ago by Webster and co-workers. ${ }^{9}$ In a comparative analysis, Guadayol and co-workers ${ }^{10}$ showed that the two former methods, if properly used, may fulfill the main characteristics for experimental studies in microcosms or mesocosms, e.g.: stationarity, homogeneity, isotropy, and turbulence intensities close to nature.

Vertically or axis-symmetrically oscillating grids have been prevalently used for biological experiments because they are quite customizable by changing the grid mesh, the diameter of the rods, the size and the shape of the grid and of the container, the frequency and the length of the stroke. All of these parameters can be measured independently and the turbulence level derived and fixed a priori, while requiring a calibration for each grid type. Guadayol and co-workers ${ }^{10}$ carried out a wide set of measurements with different grids and produced standard turbulence levels for a wide array of experimental setups.

Following a similar approach, we designed a device to produce grid generated turbulence with a computer-driven 
electromechanical system suitable to control the movement of the grids. The rationale for this effort was the need to explore variability in turbulent regimes while keeping the largest possible control on its generation.

Our device can be considered complementary to microfluidic devices, which are undergoing an impressive development. ${ }^{11}$ These are providing more and more information on mechanisms acting on single organisms or single cells or group of organisms at micro-scale, while mesocosms allow the characterization of the impact of those processes on a large numbers of organisms and their interaction across different scales.

In this paper, we describe the design of such device, TURBOGEN, a flexible generator of micro-turbulence on large fluid volumes and its main characteristics. We also report the results of the calibration experiments that were carried out with three independent methods. Most of the results will pertain to the generation of stationary, homogeneous, isotropic, low intensity turbulence. This prototype has unique features that enable also a scaling up of the instrument suitable for the generation of agitation in industrial photobioreactors and/or to work with large volumes of diluted, natural-like cultures. In the perspective of plankton studies, the motivation for this effort stems from the opportunities offered by modern biological approaches, e.g., the -omics which, for the time being, require large volumes of low concentrated cultures, for analyzing the plankton responses from single cell processes $^{12-14}$ to biological interactions. ${ }^{15-22}$ Those responses reflect the evolutionary history of plankton ${ }^{23}$ and are a key to understand their dynamics and succession in the aquatic environments. $^{24}$

\section{RESULTS}

\section{A. The apparatus}

The main characteristics of TURBOGEN (Fig. 1) are reported in Table I. In synthesis, TURBOGEN is able to produce computer-controlled customizable turbulence levels with the advantage of being repeatable with a high level of accuracy as many times as needed to characterize a specific flow regime.

The turbulence generating system (TURBOGEN, Fig. 1(a)) designed and realized by M2M Engineering (http://www.m2mengineering.it/en.html) is powered by a stepby-step bidirectional motor, which controls the vertical movement of all the grids (Figs. 1(b) and 2), whose displacement is monitored by a dedicated sensor on the central pylon (Fig. 1(a), white arrow). Unlike most existing experimental setups, which use a rotor to directly control the rod and therefore produce oscillatory velocities correlated to the motor's frequency, setup described here, TURBOGEN, controls the rod digitally and is able to produce constant non-oscillating velocities and accelerations. In this respect, TURBOGEN is similar to the system reported by Guadayol and co-workers ${ }^{10}$ used to generate turbulence in a mesocosm. The additional characteristic is that three of the key parameters for turbulence generation, i.e., the grid velocity, the acceleration, and the stroke, can be varied arbitrarily during the course of the experiment.

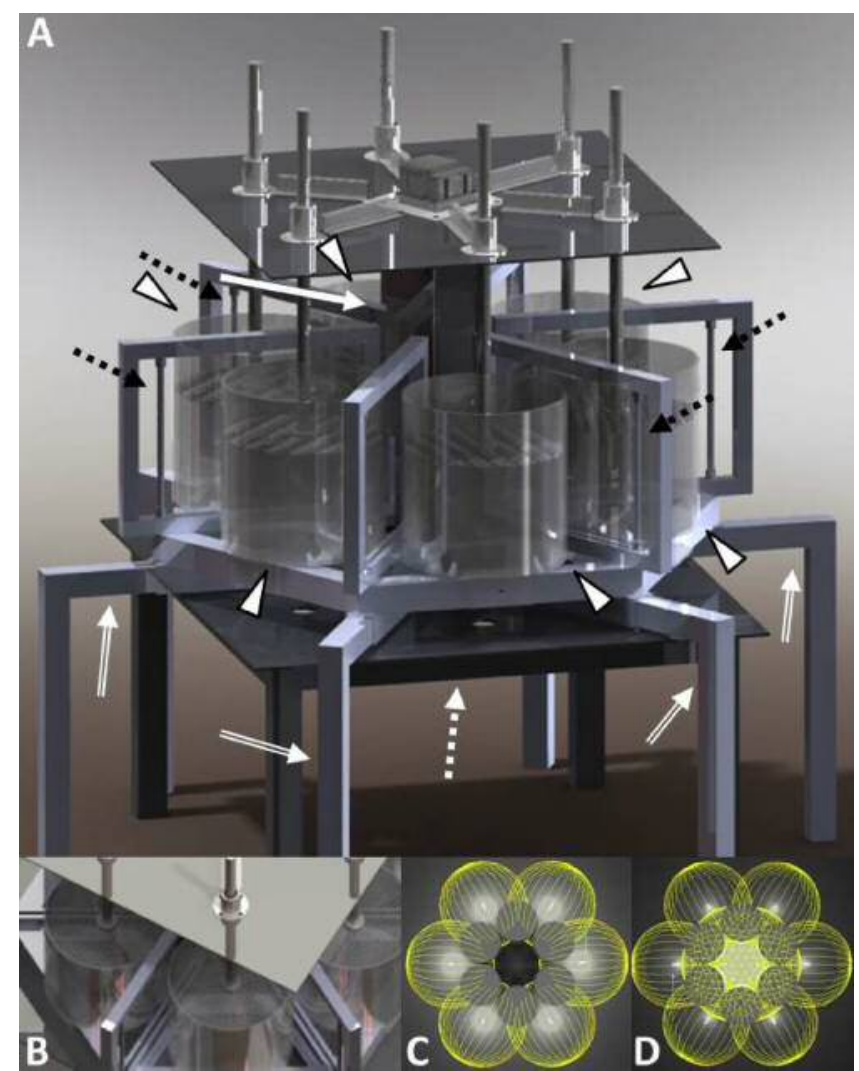

FIG. 1. TURBOGEN description. (a) TURBOGEN is composed of six Plexiglas cylinders (arrowheads) mounted on a six-legged table (double arrows). Circular square-mashed grids (see Figure 2) move up and down into the cylinders. The grids are connected via a Plexiglas stick to a plane that is in turn connected to the central pylon (white arrow) containing the motor. The central pylon lays on a separate table (white dotted arrow) that is not in physical contact with the six-legged table that holds the cylinders. Six light bulbs (black dotted arrows) are mounted on the present setup. (b) Grids are mounted on Plexiglas sticks that are connected to a plate. (c) Light field produced when TURBOGEN is equipped with 6 or (d) twelve light bulbs.

The containers are Plexiglas $®$ cylinders (height $300 \mathrm{~mm}$, base diameter $250 \mathrm{~mm}$ ) with a total capacity of 14.71 (Fig. 1(a), arrowheads) and a working volume of 13 l. Figure 2 shows details of one of the circular grids used in TURBOGEN. In particular, grids $(\varnothing 215 \mathrm{~mm})$ are composed of 10 rods (Ø $2 \mathrm{~mm})$ different in length $(2 \times 125 \mathrm{~mm}, 2 \times 170 \mathrm{~mm}$, $2 \times 195 \mathrm{~mm}, 2 \times 210 \mathrm{~mm}, 2 \times 215 \mathrm{~mm}$ ) that perpendicularly intersect 10 identical rods forming a $20 \mathrm{~mm}^{2}$ meshed grid.

The structure supporting the cylinders (Fig. 1(a), double arrows) is detached from the main structure (Fig. 1(a), white dotted arrow) in order to prevent the direct transmission of the moving pylon vibrations to the containers. For algal growth experiments, TURBOGEN is equipped with six white cold neon light bulbs (Fig. 1(a), black dotted arrows) optimized for phytoplankton. The illumination is homogeneously distributed on the containers (Fig. 1(c)) and the light-intensity can be regulated electronically or using a mobile support. If more light intensity is needed, six more light bulbs can be fitted on the same structure closer to the central pylon (Fig. 1(d)). Single containers can be shielded if experiments at different light intensities must be carried out. Each container has a small hole in the bottom, slightly out of center, for sampling or cell gathering by spilling. Operating cycles can be programmed 
TABLE I. Main characteristics of TURBOGEN and ranges of possible values.

\begin{tabular}{lcc}
\hline \hline Parameter & Range & Units \\
\hline Oscillation amplitude (stroke) & $0.1-300$ & $\mathrm{~mm}$ \\
Positioning accuracy & 0.1 & $\mathrm{~mm}$ \\
Velocity of the grids & $0-216$ & $\mathrm{~mm} \mathrm{~s}^{-1}$ \\
Acceleration of the grids & $0-1000$ & $\mathrm{~mm} \mathrm{~s}^{-2}$ \\
Oscillation frequency of the grids & $0-10$ & $\mathrm{~s}^{-1}$ \\
Time dependent velocity profile of the grids & Yes & $\mathrm{dl}$ \\
Time dependent acceleration profile of the grids & Yes & $\mathrm{dl}$ \\
Time dependent profile Stroke & Settable & $\mathrm{dl}$ \\
Initial position stroke & Settable & $\mathrm{dl}$ \\
Final position stroke & Settable & $\mathrm{dl}$ \\
Time dependent initial position stroke & Yes & $\mathrm{dl}$ \\
Time dependent final position stroke & Yes & $\mathrm{dl}$ \\
Position sensor & Yes & $\mathrm{dl}$ \\
Extra course alarm position sensor & Yes & $\mathrm{dl}$ \\
Anomaly control alarm & Yes & $\mathrm{dl}$ \\
Overstress alarm & Yes & $\mathrm{dl}$ \\
Number of cylinders & $1-6$ & $\mathrm{dl}$ \\
Capacity of each cylinder & 13 & 1 \\
Irradiance & $0-150$ & $\mu \mathrm{mol} \mathrm{photons} \mathrm{m}^{-2} \mathrm{~s}^{-1}$ \\
\hline
\end{tabular}

by dedicated software, which also records the time course of the movement performed allowing further comparison with turbulence measurements and analysis.

\section{B. Measurements}

A set of measurements aimed at the calibration of the system and at the flow regime characterization (i.e., without considering the effect of turbulence on populations) were performed. All variables and abbreviations are reported in Nomenclature. Flow measurements were performed using image analysis, in particular, the feature tracking technique $\left(\mathrm{FT}^{25}\right)$ was here considered. The FT technique provided a multi-point velocity time series, i.e., the instantaneous spatial velocity fields in the plane of measurements, in this case, $u(x, z, t), w(x, z, t)$, about 50000 images (120 s) were acquired and analyzed for each experiment. Starting from the measured velocities, the energy dissipation rate $\varepsilon$ was estimated following the different formulation reported below.

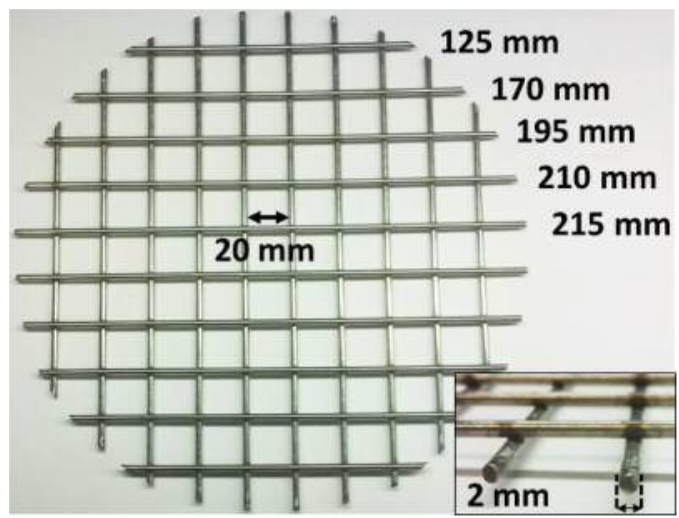

FIG. 2. Grid used in the present setup for generating turbulence. The rod sizes are reported on the right. The grid is vertically and horizontally symmetrical. Inset: lateral view of the grid, showing the thickness of each rod.
The obtained values have then been compared with the theoretical energy production $\mathrm{P}$ estimated as described below.

\section{Estimation of the theoretical dissipation/ production rate}

The estimation of the kinetic energy dissipation rate, $\varepsilon$, in oscillating systems can be performed using different approaches. ${ }^{10}$ Usually, the turbulent flow is assumed to be homogeneous and stationary; in this case, the theoretical rate of kinetic energy production, $\mathrm{P}$, must equate its dissipation, $\varepsilon$. In systems like TURBOGEN in which the grid does not follow a sinusoidal motion, the production can be estimated by the following expression: ${ }^{26}$

$$
P=\frac{1}{2} C_{d} S_{A} s^{-3} V^{-1} f\left(t_{1}^{-2}+t_{2}^{-2}\right),
$$

where $C_{d}$ is the drag coefficient set at $0.018, S_{A}$ is the solid area of the grid set at $0.24 \%$ (see Table 3, Grid 2 in Ref. 10), $s$ is the stroke length, $V$ is the volume of the container, $f$ is the oscillation frequency (Table II), and $t_{1}, t_{2}$ the time the grid takes in each displacement upwards and downwards, respectively. A list of the aforementioned parameters and of the recovered production values for each experiment is reported in Table II.

\section{Velocity gradients}

A first estimation of $\varepsilon$ was obtained according to the expression, ${ }^{27,28}$

$$
\begin{aligned}
\varepsilon_{g}= & 3 v\left[\left(\frac{d u}{d x}\right)^{2}+\left(\frac{d w}{d z}\right)^{2}+\left(\frac{d u}{d z}\right)^{2}+\left(\frac{d w}{d x}\right)^{2}\right. \\
& \left.+2\left(\frac{d u}{d z} \frac{d w}{d x}\right)+\frac{2}{3}\left(\frac{d u}{d x} \frac{d w}{d z}\right)\right],
\end{aligned}
$$

where $v$ is the kinematic viscosity. Velocity gradients were estimated from the instantaneous velocity fields by a central 
TABLE II. List of the calibration experiments, experimental conditions, and recorded parameters.

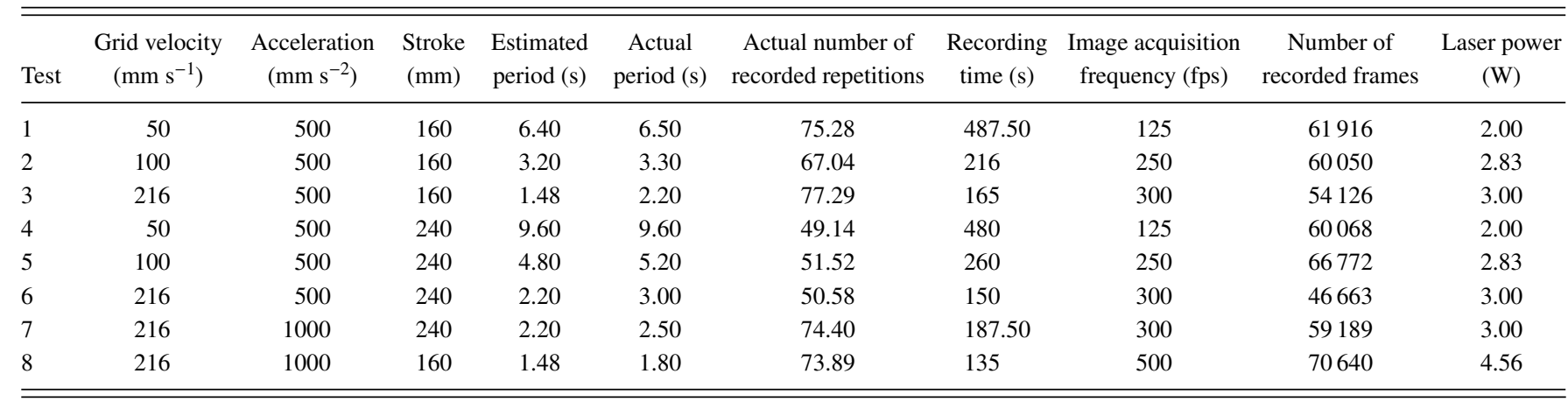

finite difference scheme and then time averaged. Figure 3(a) shows $\varepsilon_{\mathrm{g}}$ estimated for each performed experiment (Table II) as a function of $\mathrm{P} / \mathrm{C}_{\mathrm{d}}$. Since derivatives are strongly dependent upon the grid resolution, consistency of this estimation was verified by comparing the Kolmogorov length scale,

$$
\eta_{K}=\left(\frac{v^{3}}{\varepsilon}\right)^{\frac{1}{4}},
$$

with the smallest resolved scale $(2 \cdot \mathrm{mm}$ in this case). In the performed experiments, the recovered values of the Kolmogorov scales ranged from 0.4 to $1.9 \mathrm{~mm}$, i.e., about the same order of the grid resolution.

\section{Turbulent kinetic energy (TKE) decay method}

By definition, the dissipation can be estimated from the decay of TKE in time. In particular, following Peters and
Gross ${ }^{26}$ we considered the mean rate of the energy decay after the passage of the grid. TKE was estimated from the standard deviations of the horizontal and vertical velocities calculated for each cycle and then phase averaged. Typically, during a grid cycle, the energy evolution profile showed a general decay with two local inputs corresponding to the downward and upward grid movement. Also, as observed by Peters and Gross ${ }^{26}$ the decay rate followed a negative log-log trend after the onset of a peak but it was not constant over the period. In this case, since the recovered values are of the same order of magnitude, we considered the decay rate immediately after the peak. Figure 3(b) shows $\varepsilon$ values estimated with TKE decay plotted vs. $\mathrm{P} / \mathrm{C}_{\mathrm{d}}$ for each experiment performed (Table II).

\section{Energy dissipation law method}

In our experiments, we assume the existence of an inertial cascade (see Section II B 6) where energy is injected at
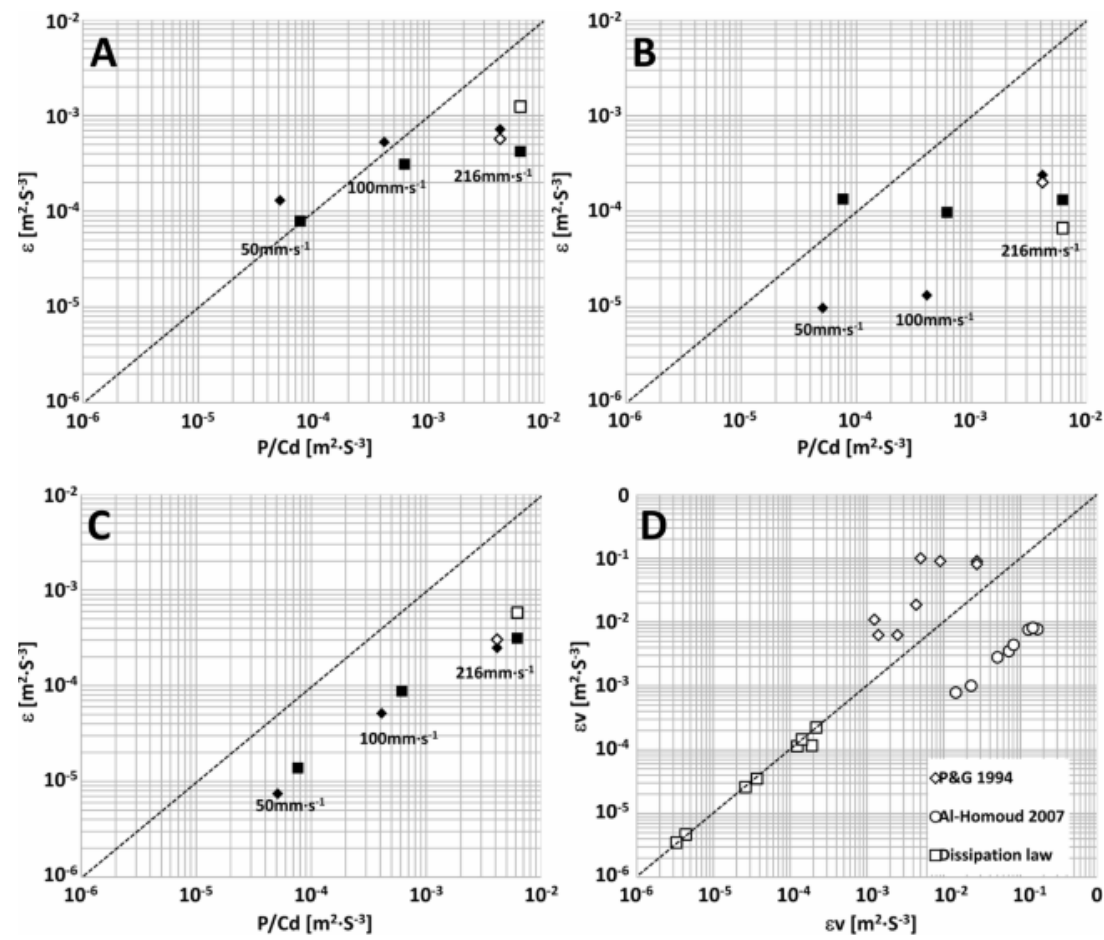

FIG. 3. Dissipation $\varepsilon$ as a function of $\mathrm{P} / \mathrm{C}_{\mathrm{d}}$, respectively, estimated from: (a) Al-Homoud and Hondzo ${ }^{27,28} \mathrm{HA}^{2}$ Eq. (2); (b) Peters and Gross ${ }^{26}$ PG; (c) Taylor ${ }^{29}$ DL, Eq. (4). Full symbols correspond to a grid acceleration $500 \mathrm{~mm} \mathrm{~s}^{-2}$; open symbols to a grid acceleration $1000 \mathrm{~mm} \mathrm{~s}^{-2}$. Squares correspond to stroke length $160 \mathrm{~mm}$; rhombs correspond to stroke length, $240 \mathrm{~mm}$. The grid speed is reported on the plot. The dotted line indicates the balance between energy production and dissipation. ${ }^{10}$ (d) Horizontal $\varepsilon_{\mathrm{ux}}$ vs vertical dissipation $\varepsilon_{\mathrm{uz}}$ (isotropy). One dimensional dissipations were, respectively, estimated from: (circles) Al-Homoud and Hondzo, ${ }^{27,28}$ (rhombs) Peters and Gross, ${ }^{26}$ (squares) Taylor. ${ }^{29}$ The dotted line ( $\varepsilon_{\mathrm{ux}}$ equals $\varepsilon_{\mathrm{uz}}$ ) corresponds to isotropy. 
large scale and is then transferred to smaller scales down to the Kolmogorov scale where energy is dissipated. The corresponding dissipation rate can therefore be estimated from the energy injection rate of TKE at the largest scales ${ }^{29}$

$$
\varepsilon_{d l}=A v_{r m s}^{3} l^{-1},
$$

where A is a universal constant assumed to be of order 1, $l$ is the characteristic size of the largest eddies, i.e., the integral scale, commonly assumed equal to the mesh size, and $v_{r m s}$ quantifies the intensity of turbulent fluctuations. In our estimation, we considered the rms of the vertical velocity, however, we verified the flow isotropy (see Sec. II B 5).

In Figure 3(c), we plotted the dissipation estimated from the dissipation law method as a function of $\mathrm{P} / \mathrm{C}_{\mathrm{d}}$.

\section{Flow isotropy}

To check for the existence of isotropic conditions, we plotted the energy dissipation estimated from the horizontal component, $\varepsilon_{\mathrm{u}_{\mathrm{x}}}$, against that from the vertical component, $\varepsilon_{\mathrm{u}_{\mathrm{Z}}}$, for all the proposed methods (Fig. 3(d)).

In all the cases, the recovered differences between the horizontal and vertical dissipation estimations were below one order of magnitude, as highlighted by the fine alignment of experimental points with the 1:1 slope. To compare the degree of isotropy with previous measurements for this kind of setups, we calculated the ratio between vertical and horizontal turbulence intensities, $\mathrm{u}_{\mathrm{z}, \mathrm{rms}} / \mathrm{u}_{\mathrm{x}, \mathrm{rms}}$, as reported in Table III.

The $\mathrm{u}_{\mathrm{z}, \mathrm{rms}} / \mathrm{u}_{\mathrm{x}, \mathrm{rms}}$ ratios confirm the existence of nearly isotropic conditions for all the tests. In the grid systems, our ratio is in the range 1.3 and 1.6, with a mean value of 1.5 . Even though our estimations include measurements within the path of the oscillating grid, we did not observe significant difference from isotropic conditions.

Time averaging vorticity over 50 grid cycles (Fig. 4), it emerges that although a slight prevalence for columnar fluid structures is visible, good isotropy with spatially stationary vortices is produced. Hence, vortices are not randomly localized but always located at the same place since the grid displacement is carefully controlled and will therefore generate at every passage the exact same flow pattern (Fig. 4).

\section{Spectral analyses}

For experiments 4-6 (Table II), the energy power spectra were computed using the set of velocity fields and the FastFourier-Transform (FFT) technique. The v-component could

TABLE III. Vertical to horizontal turbulence intensities ratio, $\mathrm{u}_{\mathrm{z}, \mathrm{rms}} / \mathrm{u}_{\mathrm{x}, \mathrm{rms}}$.

\begin{tabular}{lllll}
\hline \hline Test & $\mathrm{w}_{\mathrm{rms}}$ & $\mathrm{u}_{\mathrm{rms}}$ & Ratio & Re \\
\hline 1 & 0.74 & 0.50 & 1.47 & 240 \\
2 & 0.82 & 0.53 & 1.55 & 130 \\
3 & 0.97 & 0.71 & 1.37 & 368 \\
4 & 0.99 & 0.60 & 1.65 & 106 \\
5 & 1.03 & 0.65 & 1.59 & 202 \\
6 & 1.27 & 0.77 & 1.65 & 342 \\
\hline \hline
\end{tabular}

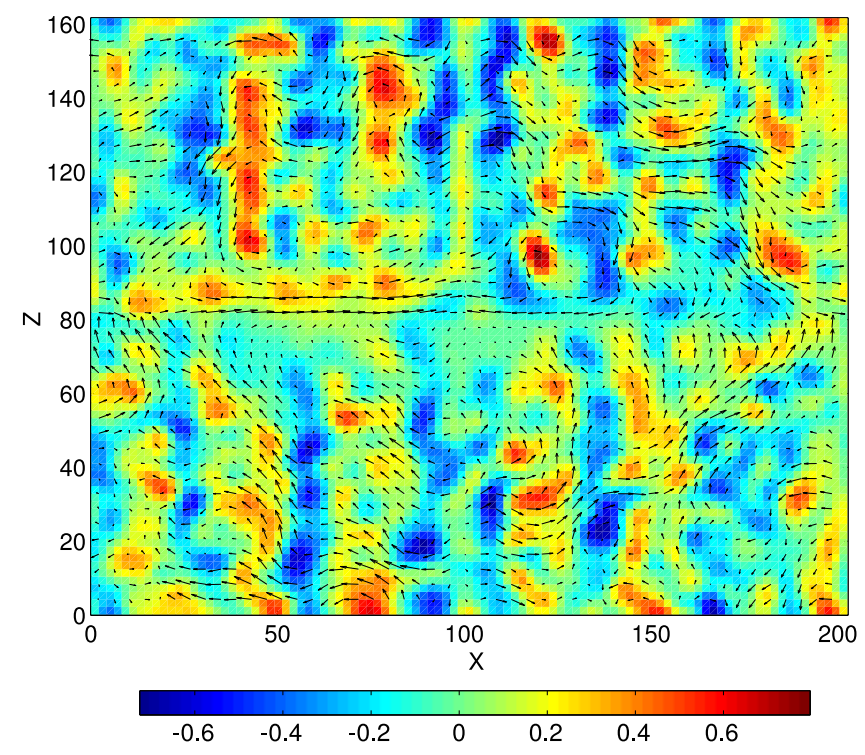

FIG. 4. Time-averaged vorticity in the case of Experiment 4 (grid velocity of $100 \mathrm{~mm} \mathrm{~s}^{-1}$ ) with quiver plot of the velocity field at scale 1 . The emergence of a pattern of time-averaged vortices highlights the stability of the flow structures from one cycle to another.

not be captured by the FFT technique; however, as the container exhibits a pure central symmetry in the horizontal section, we can assume with confidence that both horizontal velocity components are fairly identical. Theory predicts that in the inertial cascade, i.e., in the range of scales which are not directly affected by energy input, the power spectrum of energy follows:

$$
\varphi=C \varepsilon^{2 / 3} \kappa^{-5 / 3},
$$

where $\kappa=2 \pi k / \ell$ is the wavenumber associated to the physical scale $\ell / k$ and the coefficient $C$ is universal and equals to $1.5 .^{30}$ Figure 5 shows the resulting time-averaged power spectra of $u^{2}, w^{2}$, and the corresponding turbulent kinetic energy $q=\left(2 u^{2}+w^{2}\right)$, for each accessible annular wavenumber

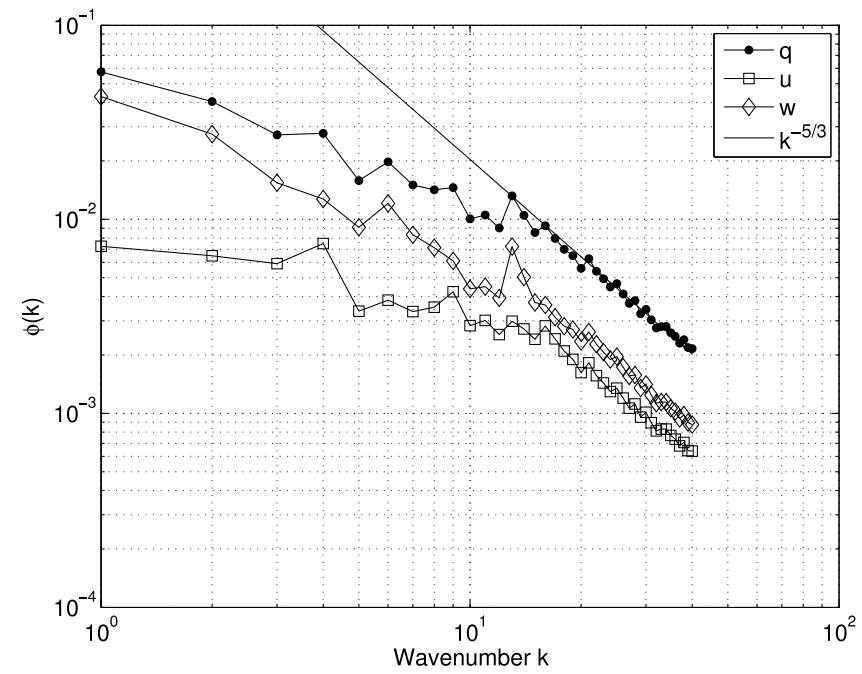

FIG. 5. Time and space averaged power spectra of the total energy $q$, horizontal velocity component $\mathrm{u}$, and vertical velocity component $\mathrm{w}$. Isotropy is recovered in the inertial cascade formed for scales smaller than the grid mesh size $(\mathrm{k}=12)$. 
$k=\left(k_{x}^{2}+k_{z}^{2}\right)^{1 / 2}$, where $k_{\mathrm{x}}$ and $k_{\mathrm{z}}$ are the wavenumbers along the $\mathrm{x}$ and $\mathrm{z}$ directions, respectively. Annular wavenumber $k$ has been normalized using the grid diameter. For the kinetic energy $q$, a small but clear peak appears at $k=12$ which corresponds to the wavenumber associated with the grid mesh size. Since the energy is injected through displacements of the grid, the inertial cascade starts only for $k>12$ and follows accurately a $-5 / 3$ slope. We can see that this peak appears on the component $w$ but not on $u$, which translates the fact that the energy input is transmitted through vertical movements. For each velocity component, a proper inertial cascade only occurs for $k>12$. Finally, it can be seen that the power spectra of $u$ are systematically lower than that of $w$, another evidence of the residual anisotropy in the system pointed out by a ratio greater than 1 .

\section{Spectral analysis along the vertical direction}

As the peak in the spectra is linked to the grid displacements, it is interesting to look how the energy distribution varies according to the position of interest in the container. We performed a series of one-dimensional spectra along the horizontal direction, performed at different vertical levels both in the laboratory (Eulerian) and grid (Lagrangian) frames of reference. To analyse the experimental setup, we used the case with $100 \mathrm{~mm} \mathrm{~s}^{-1}$ grid velocity. Figure 6 shows the various positions chosen to compute the spectra. Black lines represent the z-levels at which the $1 \mathrm{~d}$ spectra in the Lagrangian frame of reference, i.e., always at fixed distances from the

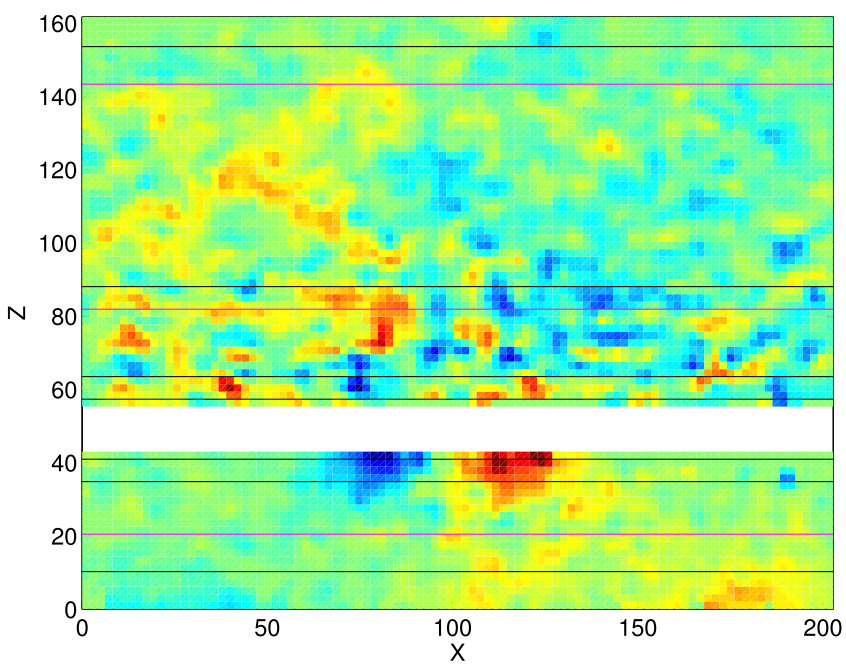

FIG. 6. 2D snapshot of the vorticity magnitude. The white rectangle corresponds to the position of the grid. In black, lines used to average properties along $\mathrm{X}$-axis at fixed distance in the grid frame of reference. In magenta, examples of line used to average properties along $\mathrm{X}$-axis at fixed distance in the container frame of reference.

grid have been conducted, and in magenta, the z-levels of the 1d spectra in the Eulerian frame of reference, i.e., always at fixed distances from the container's boundaries. As the grid moves up and down, lines defined in the Lagrangian frame of reference will appear or disappear above or below the grid according to the grid position.

We first looked at statistics in the Lagrangian frame of reference. Figure 7(a) shows the power spectra, at 4, 15,
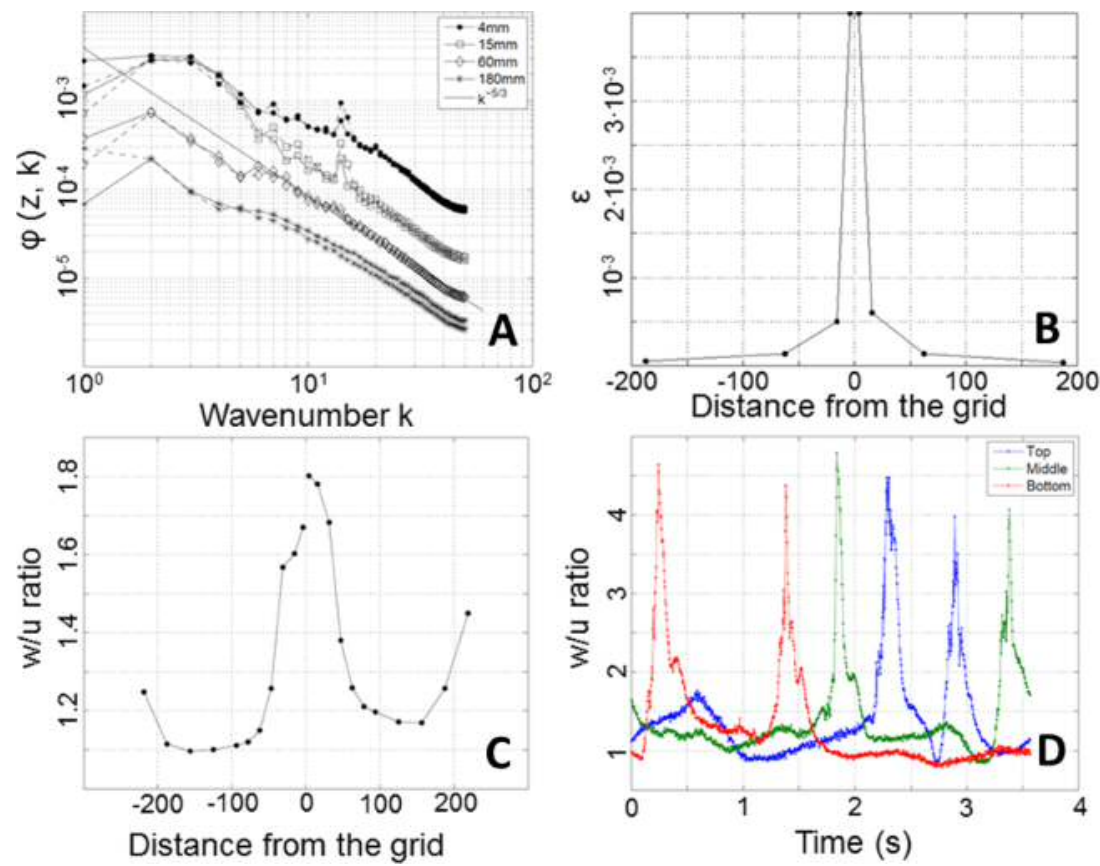

FIG. 7. Flow field characterization. (a) Time and space averaged power spectra of energy $q$ obtained at different fixed distances from the grid (black lines in Fig. 6) in the case of velocity grid of $100 \mathrm{~mm} \mathrm{~s}^{-1}$. The inertial cascade $\left(\mathrm{k}^{-5 / 3}\right)$ is well developed at all scale when far enough from the influence of the grid, e.g., at $60 \mathrm{~mm}$. (b) Time and space averaged dissipation rates at different fixed distances from the grid in the case of velocity grid of $100 \mathrm{~mm} \mathrm{~s}^{-1}$. The symmetry of dissipation with respect to the grid is well defined with high dissipate close to the grid and a quick relaxation as one move away from the grid. (c) Time and space averaged horizontal to vertical velocity component ratio at different fixed distances from the grid (black lines in Fig. 6) in the case of velocity grid of $100 \mathrm{~mm} \mathrm{~s}^{-1}$. Isotropy is almost recovered when far from the grid with a ratio down to 1.1. (d) Space averaged horizontal to vertical velocity component ratio at the bottom, middle, and top of the container. The large values of the ratio correspond to the passage of the grid in the case of velocity grid of 100 mm $\mathrm{s}^{-1}$. Relaxation time to the isotropic state is related to the grid velocity. 
60 , and $180 \mathrm{~mm}$ both above and below the grid, and their corresponding $k^{-5 / 3}$ line. Noteworthy is the quasi-perfect symmetry displayed by the energy distribution with respect to the distance from the grid, except for the position at $180 \mathrm{~mm}$ where the boundary conditions exerted by the bottom of the cylinder and the surface at the top, differently affect the power spectra. It can also be seen that, when far enough from the grid, the turbulent inertial cascade well extends with a $-5 / 3$ slope up to scales that are larger than the grid mesh size. Using Eq. (5), we get the corresponding dissipation rates for various distances from the grid, as shown in Figure 7(b). Dissipation rate is large at the vicinity of the grid $\left(\varepsilon \sim 10^{-3} \mathrm{~m}^{2} \mathrm{~s}^{-3}\right)$; however, falls quickly over two orders of magnitude $\left(\varepsilon \sim 10^{-6} \mathrm{~m}^{2} \mathrm{~s}^{-3}\right)$, as we take distance from the grid, to reach eventually values commonly found in realistic marine environments. ${ }^{3}$ Figure 7(c) shows the ratio $w_{\mathrm{rms}} / u_{\mathrm{rms}}$ for the same distances from the grid. We see that at $75 \mathrm{~mm}$ away from the grid, not only the inertial cascade is developed over almost all accessible physical scales but isotropy is produced accurately with a ratio of $w_{\mathrm{rms}} / u_{\mathrm{rms}}=1.05$ below the grid. When above the grid, the velocity ratio is slightly larger, $w_{\text {rms }} / u_{\text {rms }}=1.21$. Here, the free surface boundary condition affects more effectively than the bottom wall the velocity distribution among components.

Looking at statistics in the Eulerian frame of reference, Figure 7(d) shows the evolution of $w_{\mathrm{rms}} / u_{\mathrm{rms}}$ at fixed positions in the top, middle, and bottom of the container average over one grid cycles. The experiment can be decomposed into two time periods. First, a large wave of anisotropy (large $w_{\text {rms }} / u_{\text {rms }}$ ) is injected in the flow through the grid displacement, and second, the flow relaxes toward an isotropic state $\left(w_{\mathrm{rms}} / u_{\mathrm{rms}} \approx 1\right)$. The relaxation to isotropy time scale, $\tau_{R}$, is somewhat small and constant for all different positions, giving $\tau_{R} \approx 0.25 \mathrm{~s}$. Hence, in this experiment, with a grid cycle of $3.2 \mathrm{~s}$, the time-period of anisotropy represents around $15 \%$ of the cycle time scale. We ran this analysis for all the experiments (with grid velocity 50,100, and $216 \mathrm{~mm} \mathrm{~s}^{-1}$ ) and found that the period of anisotropy always represented $15 \%$ of the cycle time scale.

\section{DISCUSSION}

The rate of dissipation in this setup ranged from $10^{-6}$ to $10^{-3} \mathrm{~m}^{2} \mathrm{~s}^{-3}$ with the lower value falling within the range present in the natural environment. ${ }^{3}$ In agreement with Guadayol and co-workers, ${ }^{10}$ we found that the dissipation estimated within the stroke is linearly correlated to the theoretical energy production, P. Several reasons are responsible for the different estimations of $\varepsilon$ resulting from the diverse methods utilized, the main of which are likely to be the values of constants and/or coefficients in the formulae and the related approximations and the errors introduced in the calculation scheme, i.e., grid interpolation and gradients calculations. We further confirmed, consistent with Guadayol and colleagues' study, ${ }^{10}$ that the TKE decay method overestimates $\varepsilon$ respect to the dissipation law method.

TURBOGEN is among the most advanced turbulence generators based on vertically oscillating grids presently available, since the motor that powers the central pylon bearing the grids performs movements that are finely controlled with a high precision. Such movements are very smooth with no velocity variations over the stroke, unless the variation is desired. The movement generated is linear through almost the whole path of the grids since it is not based on a pulley system with rotating wheels and belts. In pulleybased turbulence generators, the movement is sinusoidal with velocity variations all over the oscillation cycle. On the contrary, TURBOGEN produces a linear movement with constant velocity. Acceleration is present only at the top and bottom of the path, where the grid direction has to change from upwards to downwards or vice-versa. Nonetheless, also this acceleration is computer-controlled and can be varied according to experimental needs. These features enable the production of a controlled, measured, and stable dissipation rate that can be repeated over different experiments. Moreover, passing through the whole container, the grid displacements impose a flow with a first wave of large anisotropy and a period of accurate isotropy. Such setup could be of use for the study of various realistic cases such as, e.g., benthic turbulence, internal wave breaking, etc.

One of the best features characterizing TURBOGEN is its versatility and the possibility to generate virtually every turbulence field characterized by very high to very low $\varepsilon$ levels. This can be done by tuning the different computer-controlled variables. The setup proposed here produces a very wide range of turbulence levels, also it has to be stressed that this range can even be widened by changing the grid mesh, solidity, or the rod size. In other setups, e.g., Couette cylinders, the possible array of turbulence levels that can be produced are narrower compared to TURBOGEN and in others, like pulley-system based oscillating grid, the grid movement is sinusoidal and not linear like in TURBOGEN and this feature is crucial to have a well-defined and repeatable turbulence field over different experiments.

The effects of turbulence on living organisms are paramount in both marine biology and ecology. For this reason, several studies have been carried out to identify such effects on a wide variety of organisms, spanning from metazoans to bacteria. The existing literature on this topic shows that most phytoplankton and microzooplankton are within a size range that should not be impacted by turbulence but are instead mostly affected by viscosity. However, it has been experimentally shown that both diatoms and dinoflagellates respond to turbulence. ${ }^{23,24,31,32}$ With its light bulbs, TURBOGEN can be easily applied to algal growth experiments. TURBOGEN is a prototype suitable for academic research but poses the bases for an industrial follow-up as well. In photobioreactors, mechanical agitation is used to assure mixing and thus to make nutrients and light available to the whole biomass growing. ${ }^{33-36}$ TURBOGEN was designed to produce micro-scale turbulence in mesocosms but scaling up this device will enhance agitation control on biomass production. The extremely high versatility of this device will make it very useful to grow algal taxa that require peculiar agitation conditions or genetically modified strains. ${ }^{37}$

As demonstrated by this study, TURBOGEN allows to reproduce turbulence fields that are close to those existing 
in real marine environment, in six 13-1 beakers at once. These properties greatly facilitate the run of biological and technical replicates that could in turn permit the study of marine turbulence using omics approaches. As an example, in transcriptomics and metabolomics, the biomass required to isolate RNA or metabolites is fairly high. The large volume of the cylinders allows to gather sufficient amount of biomass, preventing overcrowding and self-shading. Moreover, the total control of turbulent fields improves reproducibility of the experiments.

TURBOGEN will be calibrated for non-stationary turbulence fields. As stated above, grid velocity, stroke, and acceleration can be varied also during a single experiment by means of a user-friendly interface of the software that controls the motor. This would produce a turbulence field that is not homogeneous in time. Experiments run with varying features would better mimic the natural environment where turbulence inputs are not constant in time and space. Additional setups and experimental plans will be explored in the future, in order to give an exhaustive view of the range of the possible turbulence fields that TURBOGEN can produce.

In conclusion, TURBOGEN is a high throughput turbulence generator based on vertically oscillating grids able to produce isotropic micro-scale turbulence over a wide range of intensities. Calibration experiments have pointed out that TURBOGEN produces both turbulence levels that mimic those observed in the natural environment as well as higher values (possibly suitable for different applications).

\section{MATERIALS AND METHODS}

\section{A. Flow data acquisition and measurements}

A series of experiments was performed in order to analyze the flow regime with different parameter combinations (Table II).

The image acquisition device included a light diode (LD) pumped all-solid-state $5 \mathrm{~W}$ green laser (wavelength $532 \mathrm{~nm}$ ) and a high-speed digital camera (Mikrotron EoSens MC-1362, duty cycle $50 \%$, resolution $1280 \times 1024$ pixels), equipped with a $105 \mathrm{~mm}-\mathrm{f} 2.8$ objective, able to capture up to 1000 frames per second (fps, Fig. 8).

To perform velocity measurements, the fluid in the testing cylinder was seeded with neutrally buoyant hollow glass particles with an average diameter of about $30 \mu \mathrm{m}$ and a density of $1016 \mathrm{~g} \mathrm{~m}^{-3}$. The cylinder middle plane parallel to the long axis was lit by a laser light-sheet (thickness $=1.5 \mathrm{~mm}$ ). Only one plane was analyzed assuming longitudinal symmetry of the system. Images of the flow field were acquired by a charged coupled device (CCD) camera from an orthogonal view (X-Z plane in the following), the dimension of the framed area was a $204.8 \mathrm{~mm}$ (width) $\times 163.8 \mathrm{~mm}$ (height). The frame rate was varied in the range $125-500 \mathrm{fps}$ depending on the experiment (Table II).

Before starting the measurements, a calibration was performed by acquiring a reference image of a homogeneous chess-board target placed in the test section. This reference is used to calculate an individual amplification factor for each pixel, $0.16 \mathrm{~mm} \mathrm{px}^{-1}$ in our case. Based on the time

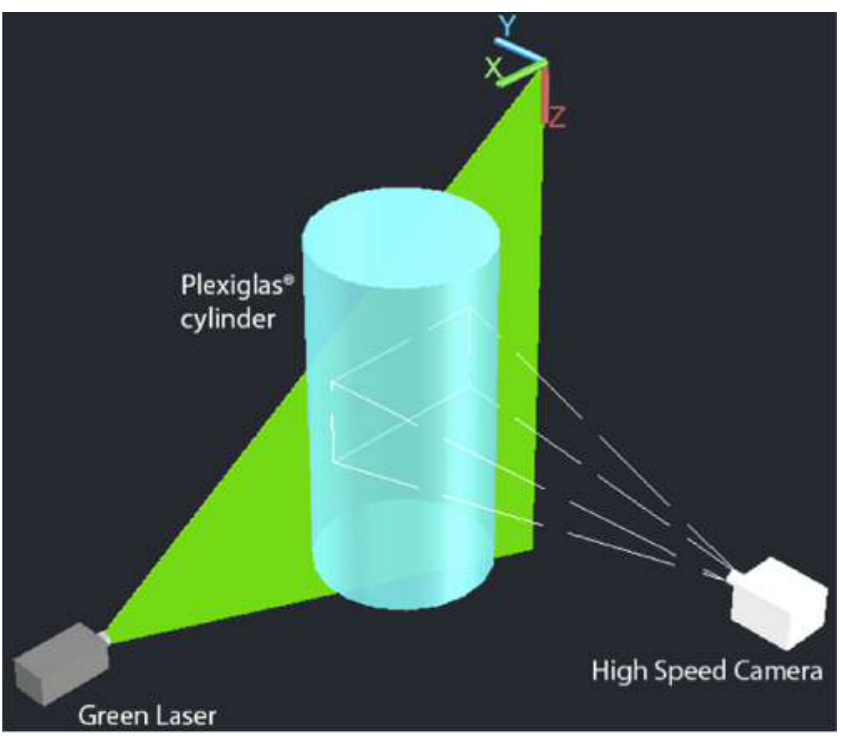

FIG. 8. Schematic view of the image acquisition system. TURBOGEN cylinder vertical (XZ) section (here indicated in blue) is lighted by a laser sheet. Images of the flow field are acquired by a high-speed videocamera located orthogonally to that view.

and spatial resolution of the video recordings, the accuracy in the measurement of the velocity can be estimated of order of $10^{-4} \mathrm{~m} \mathrm{~s}^{-1}{ }^{38}$ To improve the performance of the technique and easily detect objects in the acquired images, a preprocessing step of background subtraction and thresholding segmentation on the raw images is usually required. ${ }^{39}$ The procedure of image analysis is summarized in Figure 9.

Recorded images were post-processed using a FT algorithm that allows obtaining two components of the velocity field $\mathrm{u}_{\mathrm{x}}$ and $\mathrm{u}_{\mathrm{z}}$, respectively. The $\mathrm{FT}$ technique ${ }^{25}$ can be defined as a tracking technique based on correlation windows: displacements are evaluated considering the best correspondence in terms of a defined matching measure of selected interrogation windows between subsequent images. If we consider all surfaces inside the image to have Lambertian characters (their luminosity values do not depend on the point of view of the observer) and the illumination source to give almost constant level of light, the substantial derivative of luminosity inside the image can be considered as zero; in this way, the continuity equation for the optical flow, also called Brightness Constancy Constrain (BBC), is obtained,

$$
\frac{D I(\boldsymbol{x}, t)}{D t}=\frac{\partial I(\boldsymbol{x}, t)}{\partial t}+\frac{\partial I(\boldsymbol{x}, z)}{\partial x} u+\frac{\partial I(\boldsymbol{x}, z)}{\partial y} v=0,
$$

where $I(x, t)$ is the image intensity at the position $\mathbf{x}(x, y), t$ is the time, and $\mathrm{u}, v$ are the components of the unknown velocity vector. This law is assumed to hold for small time intervals and also in both spatial directions, typically centered on seeding particles. Since Eq. (6) is not sufficient to determine the two velocity components in each point of the field, a cost function is defined over an interrogation area $\mathrm{W}$ centered in $(\mathrm{x}, \mathrm{y})$,

$$
\zeta=\int_{W(x, y)}\left(\frac{D I}{D t}\right)^{2} d W
$$

and the velocity in $(\mathrm{x}, \mathrm{y})$ is computed as the displacement $(\mathrm{u}$, v) that minimizes $\zeta$ by imposing that its derivatives vanish, 


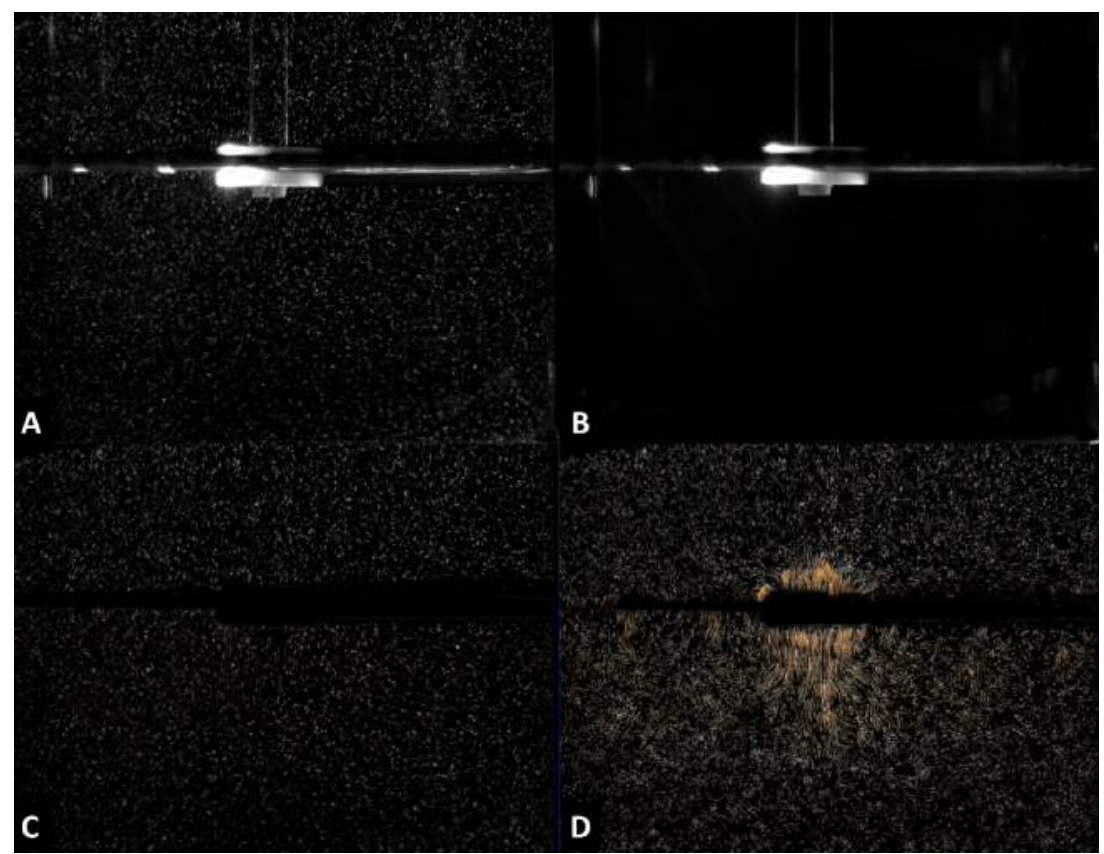

FIG. 9. Background subtraction and thresholding segmentation of acquired images. Image analysis procedure: (a) acquired, i.e., raw image; (b) after background identification; (c) image after the background subtraction; (d) set of reconstructed particle trajectories after the grid removal.

i.e., through a least square optimization. Thus, a closed set of linear equations, with unknown $\mathrm{u}$ and $\mathrm{v}$, results $\mathrm{in}^{40}$

$$
\begin{gathered}
\int_{W(x, y)}\left(\begin{array}{ll}
\left(\frac{\partial I}{\partial x}\right)^{2} & \frac{\partial I}{\partial x} \frac{\partial I}{\partial y} \\
\frac{\partial I}{\partial x} \frac{\partial I}{\partial y} & \left(\frac{\partial I}{\partial y}\right)^{2}
\end{array}\right)\left(\begin{array}{l}
u_{x} \\
u_{z}
\end{array}\right) d W \\
=\int_{W(x, y)}\left(\begin{array}{cc}
\frac{\partial I}{\partial x} & \frac{\partial I}{\partial t} \\
\frac{\partial I}{\partial y} & \frac{\partial I}{\partial t}
\end{array}\right) d W .
\end{gathered}
$$

The dimension of the interrogation window impacts on the tracking efficiency and has to be chosen according to the characteristics both of the flow and of the framed area; in this, the selected dimension was in the order of 10 pixels. It has to be noted that the interrogation windows are not distributed on a regular grid as in classical PIV (Particle Image Velocimetry) algorithms. Rather, they are located in the regions where the set of equations is well conditioned, i.e., where its eigenvalues are high enough. In other words, the problem can be solved only where a reliable solution can be found. These regions, which are characterized by high light intensity gradients in both spatial directions, are called good features to track,,$^{41}$ and in fluid flow images, they are typically centered on seeding particles. It follows that in this case, differently to classical particle tracking algorithms, no a priori consideration has to be made about the object to be tracked. ${ }^{42}$

Once the displacements in $\mathrm{x}$ and $\mathrm{z}$ directions are obtained, the Lagrangian velocities are calculated by dividing the displacement by the time interval between frames. In these experiments, at least 8000 particles have been simultaneously tracked during each cycle. By interpolating the sparse velocity vectors onto a regular grid, the Eulerian flow picture, in terms of instantaneous velocity fields and derived quantities (e.g. vorticity), is obtained as well.

\section{B. Data resampling}

In order to perform data resampling, a distance-weighted interpolation was adopted. ${ }^{43}$ For each point $\mathrm{P}_{\mathrm{g}}$ of the regular grid, the corresponding set $P_{j}(j=1,2, \ldots, J)$ of tracked features' positions placed within a certain distance from $P_{g}$ was built. Considering the squared distance from $P_{j}$ to $P_{g}, D_{j}^{2}$, and its average $\overline{D^{2}}$ over all $j$, two coefficients are introduced,

$$
\begin{gathered}
\alpha_{j}=\exp \left(\frac{-D_{j}^{2}}{\overline{D^{2}}}\right) \\
\beta_{j}=\frac{\alpha_{j}}{\sum_{j=1}^{J} \alpha_{j j}},
\end{gathered}
$$

where $0<\left(\alpha_{\mathrm{j}}, \beta_{\mathrm{j}}\right) \leq 1$ and $\sum_{\mathrm{j}=1}^{\mathrm{J}} \beta_{\mathrm{j}}=1$. The interpolated velocity vector in $P_{g}$ is then computed as

$$
u_{g}=\sum_{j=1}^{J} \beta_{j} u_{j}
$$

being vector $\mathbf{u}_{j}$ the velocity in $P_{j}$. To run this method, one has to define a minimum number of points belonging to the set $\mathrm{P}_{\mathrm{j}}$ and a maximum value for the search distance from $\mathrm{P}_{\mathrm{g}}$. In this implementation, the smallest region of research considered was a circular region of three pixels in radius surrounding each grid point. If the recovered feature number $\mathrm{J}$, in that region is lower than a fixed minimum (namely, 3), the radius of the region is increased by 1 pixel and a new search procedure is started. If the maximum value of 20 pixels for the region was reached, no value was assigned to the grid point. 


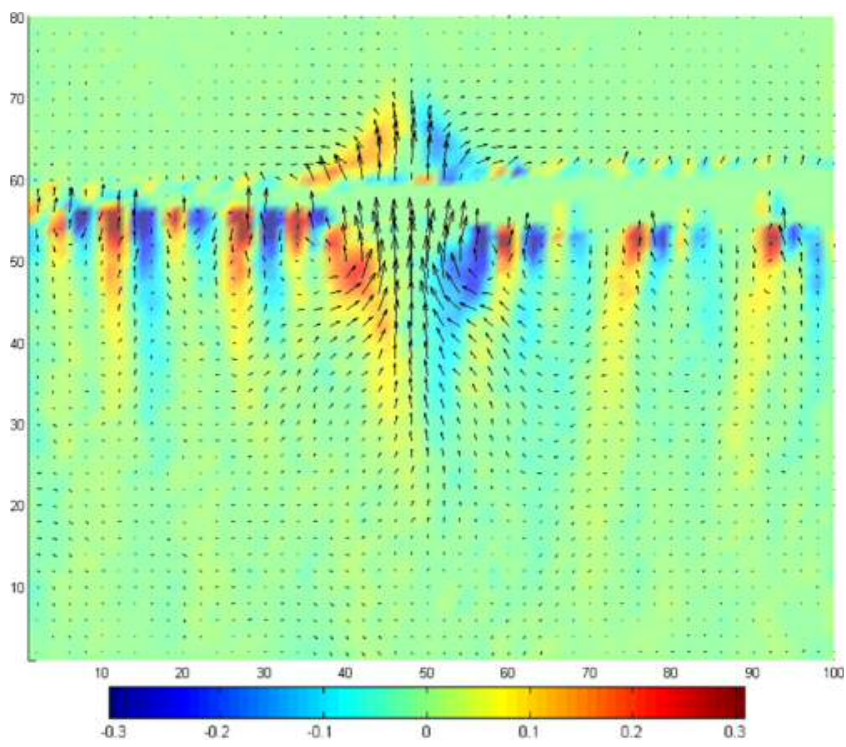

FIG. 10. An instantaneous velocity (black arrows)-vorticity (color map) field for experiment 1 . Colorbar is $\mathrm{s}^{-1}$.

Interpolation of the velocity vectors over a regular grid yielded the time evolution of the Eulerian instantaneous velocity field as a function of time; here, the grid resolution chosen for all the experiments was $80 \times 100$ grid points. The resulting temporal and spatial resolutions of the data set were 2-4 ms (depending on the acquisition frequency) and $2 \mathrm{~mm}$, respectively. Figure 10 shows an instantaneous velocity-vorticity field for experiment 1 .

Velocity gradients, kinetic energy, and dissipation can then be computed; mean $\nabla \mathrm{u}_{\mathrm{x}}, \nabla \mathrm{u}_{\mathrm{z}}$, and fluctuating $\left(\mathrm{u}_{\mathrm{x}}{ }^{\prime}, \mathrm{u}_{\mathrm{z}}{ }^{\prime}\right)$ quantities are calculated considering the phase average over the number of cycles.

\section{ACKNOWLEDGMENTS}

Rachel Macmasters is acknowledged for language check. A.A., M.I.F., D.I., M.R.d'A., and R.W. thank the Flagship project RITMARE-The Italian Research for the Sea Programme (Ricerca ITaliana per il MARE) for partial support. A.A. was funded by the European Union under FP7-People-GA No. 600407.

\section{NOMENCLATURE}

A $=$ a universal constant assumed to be of order 1

$C=$ universal constant assumed to be equal to $1.5^{30}$

$C_{d} \quad=$ drag coefficient set at $0.018^{10}$

$f \quad=$ oscillation frequency

$\mathrm{I}(\mathbf{x}, \mathrm{t})=$ image intensity at the position $\mathbf{x}(\mathrm{x}, \mathrm{y})$

$k \quad=$ wavenumber

$l=$ characteristic size of the largest eddies

$\mathrm{P} \quad=$ theoretical rate of kinetic energy production

$\mathrm{P}_{\mathrm{g}} \quad$ = each point of the grid

$\mathrm{P}_{\mathrm{j}} \quad=$ set of tracked features' positions placed within a certain distance $D_{j}$ from $P_{g}$

$q \quad=$ turbulent kinetic energy

$s \quad=$ stroke length of the grid
$S_{A} \quad=$ solid area of the grid set at $0.24 \%^{10}$

$t_{1} \quad=$ time the grid takes in each displacement upwards

$t_{2}=$ time the grid takes in each displacement downwards

$V \quad=$ volume of the container

$v \quad=$ components of the unknown velocity vector

$\mathrm{W}=$ interrogation area centered in $(\mathrm{x}, \mathrm{y})$

$\varepsilon \quad=$ kinetic energy dissipation rate

$\varepsilon_{\mathrm{g}} \quad=$ direct estimation of the kinetic energy dissipation rate $^{27,28}$

$\zeta=$ cost function defined over an interrogation area $\mathrm{W}$

$\eta_{\mathrm{k}} \quad=$ Kolmogorov scale

$\kappa=2 \pi k / \ell$ is the wavenumber associated to the physical scale $\ell / k$

$v \quad=$ kinematic viscosity

$\varphi \quad=$ power spectrum of energy

$\tau_{R}=$ relaxation to isotropy timescale

$\mathrm{BBC}=$ Brightness Constancy Constrain

$\mathrm{FT}^{25}=$ Feature Tracking

FFT $=$ Fast-Fourier-Transform

PIV = Particle Image Velocimetry

TKE $=$ Turbulent Kinetic Energy

${ }^{1}$ F. Ballantyne IV, O. M. E. Schofield, and S. A. Levin, Sci. Mar. 75, 719 (2011).

${ }^{2}$ F. Peters and J. M. Redondo, Sci. Mar. 61, 205 (1997).

${ }^{3}$ I. Lozovatsky, M. Figueroa, E. Roget, H. J. S. Fernando, and S. Shapovalov, J. Geophys. Res. 110, C05013, doi:10.1029/2004jc002708 (2005).

${ }^{4}$ F. Peters and C. Marrasé, Mar. Ecol.: Prog. Ser. 205, 291 (2002).

${ }^{5}$ L. Seuront, Mar. Ecol.: Prog. Ser. 302, 93 (2005).

${ }^{6}$ H. Yamazaki, J. G. Mitchell, L. Seuront, F. Wolk, and H. Li, Geophys. Res. Lett. 33, L01603, doi:10.1029/2005g1024103 (2006).

${ }^{7}$ M. J. Doubell, J. C. Prairie, and H. Yamazaki, Deep Sea Res., Part II 101, 207 (2014).

${ }^{8} \mathrm{~S}$. A. Thorpe, An Introduction to Ocean Turbulence (Cambridge University Press, 2007).

${ }^{9}$ D. R. Webster, A. Brathwaite, and J. Yen, Limnol. Oceanogr.: Methods 2, 1 (2004).

${ }^{10}$ Ò. Guadayol, F. Peters, J. E. Stiansen, C. Marras, and A. Lohrmann, Limnol. Oceanogr.: Methods 7, 287 (2009).

${ }^{11}$ R. Rusconi, J. S. Guasto, and R. Stocker, Nat. Phys. 10, 212 (2014).

${ }^{12}$ L. Karp-Boss, E. Boss, and P. A. Jumars, Oceanogr. Mar. Biol. 34, 71 (1996).

${ }^{13}$ M. Hondzo and A. Wüest, Environ. Sci. Technol. 43, 764 (2008).

${ }^{14}$ A. Rogato, A. Amato, D. Iudicone, M. Chiurazzi, M. I. Ferrante, and M. Ribera d'Alcalà, Mar. Genomics 24(Part 1), 95 (2015).

${ }^{15}$ B. J. Rothschild and T. R. Osborn, J. Plankton Res. 10, 465 (1988).

${ }^{16}$ J. Shimeta, P. A. Jumars, and E. J. Lessard, Limnol. Oceanogr. 40, 845 (1995).

${ }^{17}$ F. Peters, C. Marrasé, J. M. Gasol, M. M. Sala, and L. Arin, Mar. Ecol.: Prog. Ser. 172, 293 (1998).

${ }^{18}$ A. W. Visser and G. A. Jackson, Mar. Ecol.: Prog. Ser. 283, 55 (2004).

${ }^{19}$ P. Mariani, V. Botte, and M. Ribera d'Alcalà, J. Mar. Syst. 70, 273 (2008).

${ }^{20}$ C. H. Lee, H. U. Dahms, S. H. Cheng, S. Souissi, F. G. Schmitt, R. Kumar, and J. S. Hwang, Mar. Biol. 158, 1085 (2011).

${ }^{21}$ W. M. Durham, E. Climent, M. Barry, F. De Lillo, G. Boffetta, M. Cencini, and R. Stocker, Nat. Commun. 4, 2148 (2013).

${ }^{22}$ L. Seuront and H. E. Stanley, Proc. Natl. Acad. Sci. U. S. A. 111, 2206 (2014).

${ }^{23}$ R. Margalef, Oceanol. Acta 1, 493 (1978).

${ }^{24}$ S. J. Clarson, M. Steinitz-Kannan, S. V. Patwardhan, R. Kannan, R. Hartig, L. Schloesser, D. W. Hamilton, J. K. Fusaro, and R. Beltz, Silicon 1, 79 (2009).

${ }^{25}$ A. Cenedese, Z. D. Prete, M. Miozzi, and G. Querzoli, Exp. Fluids 17, 719 (2005).

${ }^{26}$ F. Peters and T. Gross, Mar. Ecol.: Prog. Ser. 115, 299 (1994).

${ }^{27}$ P. Doron, L. Bertuccioli, J. Katz, and T. R. Osborn, J. Phys. Oceanogr. 31, $2108(2001)$

${ }^{28}$ A. Al-Homoud and M. Hondzo, Environ. Fluid Mech. 7, 143 (2007).

${ }^{29}$ G. I. Taylor, Proc. R. Soc. A 151, 421 (1935). 
${ }^{30}$ S. B. Pope, Turbulent Flows (Cambridge University Press, Cambridge, 2000).

${ }^{31}$ R. Margalef, Sci. Mar. 61, 109 (1997).

${ }^{32}$ G. Llaveria, R. I. Figueroa, E. Garcés, and E. Berdalet, J. Phycol. 45, 1106 (2009).

${ }^{33}$ C. U. Ugwu, H. Aoyagi, and H. Uchiyama, Bioresour. Technol. 99, 4021 (2008).

${ }^{34}$ C. J. Hulatt and D. N. Thomas, Bioresour. Technol. 102, 5775 (2011).

${ }^{35}$ J. B. Shurin, R. L. Abbott, M. S. Deal, G. T. Kwan, E. Litchman, R. C. McBride, S. Mandal, and V. H. Smith, Ecol. Lett. 16, 1393 (2013).

${ }^{36}$ J. Huang, Y. Li, M. Wan, Y. Yan, F. Feng, X. Qu, J. Wang, G. Shen, W. Li, J. Fan, and W. Wang, Bioresour. Technol. 159, 8 (2014).
${ }^{37}$ F. Daboussi, S. Leduc, A. Maréchal, G. Dubois, V. Guyot, C. Perez-Michaut, A. Amato, A. Falciatore, A. Juillerat, M. Beurdeley, D. F. Voytas, L. Cavarec, and P. Duchateau, Nat. Commun. 5, 3831 (2014).

${ }^{38}$ M. Miozzi, B. Jacob, and A. Olivieri, Exp. Fluids 45, 765 (2008).

${ }^{39}$ E. A. Cowen and S. G. Monismith, Exp. Fluids 22, 199 (1997).

${ }^{40}$ C. Tomasi and T. Kanade, Proc. Natl. Acad. Sci. U. S. A. 90, 9795 (1993).

${ }^{41}$ J. Shi and C. Tomasi, "Good features to track," in Proceedings of the 1994 IEEE Conference on Computer Vision and Pattern Recognition (CVPR), IEEE Computer Society, Los Alamitos: Seattle, WA (IEEE, 1994), p. 593.

${ }^{42}$ M. Moroni and A. Cenedese, Meas. Sci. Technol. 16, 2307 (2005).

${ }^{43}$ D. Chetverikov, Int. J. Pattern Recognit. Artif. Intell. 17, 487 (2003). 